\title{
BUCKLING OF AN ELASTIC MEMBRANE ON A SURFACE OF REVOLUTION*
}

$\mathrm{By}$

\author{
MURRAY SCHECHTER \\ Lehigh University
}

\begin{abstract}
This paper is concerned with a generalization of the following problem in nonlinear membrane theory studied by S. Lubkin [1] and C. Sensenig [2]: a membrane in the shape of an annulus is deformed by the application of a hydrostatic pressure to the outer boundary while the inner boundary remains stress free. The membrane is constrained to lie between two lubricated plates, so that no work is done at these surfaces during the deformation. The deformation is rotationally symmetric, each point on the membrane moving along a radius so that the deformed membrane is still in the shape of an annulus. What is to be determined is the stability of this deformation with respect to asymmetric disturbances satisfying the same stress conditions at the two boundaries and the same constraint imposed by the plates.

In this paper their problem is generalized by constraining the membrane to lie on an arbitrary surface of revolution rather than a plane. Perturbation theory is used to determine instabilities, as in Sensenig's solution of the plane problem. It does not appear in general possible to determine even the symmetric deformation explicitly (as opposed to numerically), leave alone buckling values of any appropriate parameter. The symmetric deformation when the surface is a cone, may however be explicitly determined and is the same as the symmetric deformation for an appropriate plane annulus. Furthermore, the symmetric deformation in the general case will be shown to have certain properties, which bear an interesting relation to a result of $\mathrm{F}$. John [3].

In the absence of an explicit solution to the stability problem, a numerical method for its solution has been devised and carried through on the IBM 7090 at New York University for a sphere of varying radius and also for a cone of varying half angle. The results in the limiting case of plane surfaces were checked against the explicit formula in [2].
\end{abstract}

1. Problem and methods of solution. Considering an elastic membrane lying on the surface of revolution $z=h(r)$. The membrane initially occupies the part of the surface $a \leq r \leq b$ or, equivalently, $h(a) \leq z \leq h(b)$ (Fig. 1).

We consider first a rotationally symmetric deformation of the membrane, in which it is constrained to lie on the surface. The surface is assumed to be lubricated. The symmetry of the deformation together with the fact that the membrane stays on the surface imply that the deformation is of the form

$$
(r, \theta, h(r)) \rightarrow\left(\rho_{0}(r), \theta+c, h\left(\rho_{0}\right)\right),
$$

where $c$ is a constant. We take $c=0$. Furthermore, since for any point on the membrane the first coordinate determines the third, we will hereafter write the symmetric deformation in the form $(r, \theta) \rightarrow\left(\rho_{0}, \theta\right)$.

To determine $\rho_{0}$, boundary conditions must be imposed. We require that the stress is zero on the boundary $r=a$, and that the boundary $r=b$ is subject to hydrostatic pressure $p$.

\footnotetext{
*Received March 10, 1964; revised manuscript received July 1, 1964.
} 


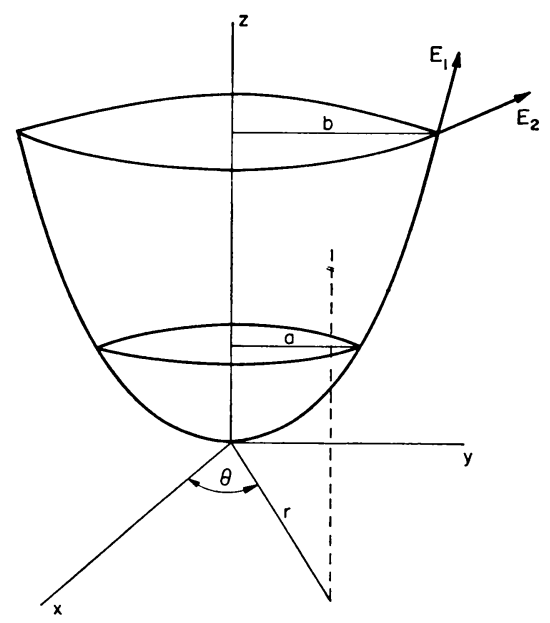

FIG. 1.

Describing the method for computing $\rho_{0}$ and determining critical pressures, we begin by applying the principle of virtual work to an arbitrary deformation

$$
(r, \theta) \rightarrow(\rho(r, \theta), \phi(r, \theta))
$$

that is subject to the same boundary conditions as the symmetric deformation ( $\rho$ and $\phi$ have period $2 \pi$ in $\theta$ ). This yields the equations

$$
D(\rho, \phi)=0, \quad(1.1 \mathrm{a}) ; \quad E(\rho, \phi)=0,
$$

where $D$ and $E$ are second-order partial differential operators. The principle of virtual work furnishes four boundary conditions

$$
B_{i}(a, b, p, \rho(a, \theta), \rho(b, \theta), \phi(a, \theta), \phi(b, \theta))=0 \quad i=1, \cdots 4 .
$$

These equations and boundary conditions may be specialized to give the differential equation and boundary conditions determining the symmetric deformation. Let

$$
\rho(\theta, r)=\rho_{0}(r), \quad(1.3) ; \quad \phi(\theta, r)=\theta .
$$

This substitution transforms the partial differential equations into ordinary differential equations, one of which is an identity. Moreover, two of the boundary conditions become identities. We are thus left with one second-order ordinary differential equation and a pair of boundary conditions for the determination of $\rho_{0}$.

Next we suppose that for some $p$ there exists a pair of functions $\eta(r, \theta)$ and $\psi(r, \theta)$ such that for all sufficiently small values of $\epsilon$

$$
\begin{aligned}
& \rho(r, \theta)=\rho_{0}(r)+\epsilon \eta(r, \theta), \\
& \phi(r, \theta)=\theta+\epsilon \psi(r, \theta)
\end{aligned}
$$

satisfy the differential equations (1.1) and the boundary conditions (1.2); i.e., we suppose that bifurcation occurs at some $p$. We may then differentiate equations (1.1) and (1.2) with respect to $\epsilon$, obtaining 


$$
\begin{aligned}
\left.\frac{\partial}{\partial \epsilon} D(\rho, \phi)\right|_{\epsilon=0} & =0, \\
\left.\frac{\partial}{\partial \epsilon} E(\rho, \phi)\right|_{\epsilon=0} & =0, \\
\left.\frac{\partial}{\partial \epsilon} B_{i}\right|_{\epsilon=0} & =0, \quad i=1, \cdots 4 .
\end{aligned}
$$

We thus have a linear second-order system of partial differential equations for $\eta$ and $\psi$, together with linear boundary conditions.

We seek $\eta$ and $\psi$ of the form

$$
\begin{aligned}
& \eta(r, \theta)=a_{0}(r)+\sum_{1}^{\infty}\left[a_{n}(r) \cos n \theta-a_{n}^{*}(r) \sin n \theta\right] \\
& \psi(r, \theta)=b_{0}(r)+\sum_{1}^{\infty}\left[b_{n}(r) \cos n \theta+b_{n}^{*}(r) \sin n \theta\right]
\end{aligned}
$$

(This choice of signs for the Fourier coefficients leads to greater symmetry in later equations.) Substituting these expansions into (1.7), (1.8), and equating the coefficients of $\sin n \theta$ and $\cos n \theta$ to zero, we get, for each $n$, a system of ordinary differential equations for $a_{n}$ and $b_{n}^{*}$ and an identical system for $a_{n}^{*}$ and $b_{n}$. Instead of the complete Fourier expansions (1.5) we thus might have, without loss of generality, sought $\eta$ and $\psi$ of the form

$$
\begin{aligned}
& \eta(r, \theta)=-x(r) \sin n \theta \\
& \psi(r, \theta)=y(r) \cos n \theta
\end{aligned}
$$

$x$ and $y$ then satisfy a pair of linear second-order differential equations together with four linear homogeneous boundary conditions. The pressure $p$ appears explicitly in the boundary conditions and implicitly in the coefficients in the differential equations for $x(r)$ and $y(r)$. The value of $p$ for which this system has a nontrivial solution is the $n$th critical pressure.

2. The strain energy and equilibrium equations. We now turn to the expression for the strain energy in terms of $\rho, \phi$, and their derivatives. We are using the standard strain energy density

$$
W=\frac{1}{2} \lambda\left(\epsilon_{1}+\epsilon_{2}\right)^{2}+\mu\left(\epsilon_{1}^{2}+\epsilon_{2}^{2}\right),
$$

where $\lambda$ and $\mu$ are the Lamé constants of the membrane and $\epsilon_{1}$ and $\epsilon_{2}$ are the principle strains; i.e.,

$$
\epsilon_{i}=\left(e_{i}\right)^{1 / 2}-1, \quad i=1,2,
$$

where $e_{1}$ and $e_{2}$ are the eigenvalues of the matrix

$$
S=\left(\begin{array}{cc}
\frac{\rho^{2} \phi_{r}^{2}+\rho_{r}^{2} g^{2}(\rho)}{g^{2}(r)} & \frac{\rho^{2} \phi_{r} \phi_{\theta}+\rho_{r} \rho_{\theta} g^{2}(\rho)}{r g(r)} \\
\frac{\rho^{2} \phi_{r} \phi_{\theta}+\rho_{r} \rho_{\theta} g^{2}(\rho)}{r g(r)} & \frac{\rho^{2} \phi_{\theta}^{2}+\rho_{\theta}^{2} g^{2}(\rho)}{r^{2}}
\end{array}\right)
$$

with 


$$
g(r)=\left[1+h^{\prime 2}(r)\right]^{1 / 2} .
$$

The last four equations give $W$ in terms of $\rho, \phi$ and their partial derivatives. For later use, we need explicit expressions for the first and second partial derivatives of $W$ with respect to $\rho, \rho_{r}, \rho_{\theta}, \phi_{r}$ and $\phi_{\theta}$ evaluated at $\rho(r, \theta)=\rho_{0}(r), \phi(r, \theta)=\theta$. The expressions for these derivatives are given in Appendix 1. In calculating these derivatives, we assume that the surface is convex; otherwise, somewhat different expressions result.

We now derive, by the principle of virtual work, the equations of equilibrium for an arbitrary deformation. Consider a deformation $(r, \theta) \rightarrow(\rho(r, \theta), \phi(r, \theta))$. The strain energy $U$ of this deformation is given by

$$
U=\int_{0}^{2 \pi} \int_{a}^{b} W\left(r, \rho, \rho_{r}, \rho_{\theta}, \phi_{r}, \phi_{\theta}\right) r g(r) d r d \theta .
$$

Let virtual displacements $\delta \rho(r, \theta)$ and $\delta \phi(r, \theta)$ be imposed upon $\rho$ and $\phi$. The expression for the variation of $U, \delta U$, may be transformed by integrating by parts and using Green's theorem. Further simplifying the result by observing that $\delta \rho$ as well as the arguments of $W$ have period $2 \pi$ in $\theta$, we find

$$
\begin{aligned}
\delta U=\int_{0}^{2 \pi} \int_{a}^{b}\left\{\delta \rho \left[r g(r) W_{\rho}-\frac{\partial}{\partial r}(r g(r)\right.\right. & \left.\left.W_{\rho_{r}}\right)-\frac{\partial}{\partial \theta}\left(r g(r) W_{\rho \theta}\right)\right] \\
& \left.-\delta \phi\left[\frac{\partial}{\partial r}\left(r g(r) W_{\phi_{r}}\right)+\frac{\partial}{\partial \theta}\left(r g(r) W_{\phi_{\theta}}\right)\right]\right\} d r d \theta \\
& +\left.\int_{0}^{2 \pi}\left[\delta \rho r g(r) W_{\rho_{r}}+\delta \phi r g(r) W_{\phi_{r}}\right] d \theta\right|_{a} ^{b}
\end{aligned}
$$

Next, we must compute the virtual work done by the pressure $p$. This computation may be facilitated by considering two unit vectors $E_{1}$ and $E_{2}$ in the tangent plane to the surface $z=h(r), E_{1}$ being tangent to the meridian curve and $E_{2}$ being perpendicular to $E_{1}$ and in the direction of increasing $\theta$ (Fig. 1). Also, let $N$ be the unit normal to the boundary of the deformed membrane in the tangent plane to the surface. In terms of $E_{1}$ and $E_{2}$ the vector virtual displacement is

$$
g(\rho) \delta \rho E_{1}(\rho, \phi)+\rho \delta \phi E_{2}(\rho, \phi) .
$$

A simple calculation shows that

$$
N=\frac{\rho_{\theta} g(\rho) E_{2}-\rho \phi_{\theta} E_{1}}{\rho^{2} \phi_{\theta}^{2}+\rho_{\theta}^{2} g^{2}(\rho)}
$$

The virtual work is given by

$$
p \int N \cdot\left[g(\rho) \delta \rho E_{1}+\rho \delta \phi E_{2}\right] d s=p \int_{0}^{2 \pi}\left[\rho \rho_{\theta} g(\rho) \delta \phi-\rho \phi_{\theta} g(\rho) \delta \rho\right] d \theta .
$$

Equating the virtual work with $\delta U$, and using the fact that $\delta \rho$ and $\delta \phi$ are arbitrary, we get the equations of equilibrium

$$
\begin{gathered}
r g(r) W_{\rho}-\frac{\partial}{\partial r}\left[r g(r) W_{\rho_{r}}\right]-\frac{\partial}{\partial \theta}\left[r g(r) W_{\rho_{\theta}}\right]=0, \\
\frac{\partial}{\partial r}\left[r g(r) W_{\phi_{r}}\right]+\frac{\partial}{\partial \theta}\left[r g(r) W_{\phi_{\theta}}\right]=0,
\end{gathered}
$$


and the boundary conditions

$$
\left.\begin{array}{l}
\left.\begin{array}{l}
r g(r) W_{\rho_{r}}=-p \rho \phi_{\theta} g(\rho) \\
r g(r) W_{\phi_{r}}=p \rho \rho_{\theta} g(\rho)
\end{array}\right\} \quad \text { at } r=b, \\
r g(r) W_{\rho_{r}}=0 \\
r g(r) W_{\phi_{r}}=0
\end{array}\right\} \quad \text { at } r=a .
$$

These are more explicit forms of (1.1) and (1.2).

3. The symmetric deformation. We now specialize the equilibrium equations to the symmetric case in accordance with (1.3) and (1.4). If $x$ is any one of the arguments of $W$, let $\partial W / \partial x$ evaluated for the symmetric deformation be denoted by $W_{x}^{0}$. Substituting the expressions for these derivatives, as given in the appendix, into (2.5), we find that $(2.5 \mathrm{~b})$ is identically satisfied and (2.5a) gives the equation

$$
\rho_{0}^{\prime \prime}\left[r g_{0}^{2}\right]+\left(\rho_{0}^{\prime}\right)^{2}\left[r g_{0} g_{0}^{\prime}\right]+\rho_{0}^{\prime}\left[g_{0}^{2}\left(1-\frac{r g^{\prime}(r)}{g(r)}\right)\right]-\frac{\rho_{0}}{r} g^{2}(r)+2 \alpha g(r)\left[g(r)-g_{0}\right]=0 \text {, }
$$

where

$$
g_{0}=g\left(\rho_{0}\right), \quad \alpha=\frac{\lambda+\mu}{\lambda+2 \mu}
$$

Of the four boundary conditions (2.6) two are vacuously satisfied and the remaining two prescribe, at $r=a$ and $r=b$, the value of the expression

$$
T(r)=-\frac{r g(r) W_{\rho_{r}}^{0}}{\left(\rho_{0}(r) g\left(\rho_{0}(r)\right)\right.}=\frac{r}{\rho_{0}} 2(\mu+\lambda)-\lambda-(\lambda+2 \mu) \rho_{0}^{\prime} \frac{r g_{0}}{\rho_{0} g(r)} .
$$

Explicitly, $T(a)=0$ and $T(b)=p$. Since $b$ is arbitrary, we may conclude that $T(r)$ represents the stress force at any point on the membrane.

Equations (3.1) and (3.2) may be thought of as a system of equations for the deformation $\rho_{0}(r)$ and the stress force $T(r)$. We may simplify this system by introducing the new variable

$$
V(r)=\frac{T-2 \mu}{\mu+2 \mu} \rho_{0}(r) .
$$

With this new variable the system (15) and (16) becomes

$$
\rho_{0}^{\prime}=\frac{g(r)}{g_{0}}\left[2 \alpha-\frac{\rho_{0}+V}{r}\right], \quad V^{\prime}=\frac{V}{r} \frac{g(r)}{g_{0}},
$$

where $g_{0}=g\left(\rho_{0}(r)\right)$.

It does not appear possible to reduce this system to quadratures except when $g(r)$ is constant. Since $g(r)=\left[1+h^{\prime 2}(r)\right]^{1 / 2}$, this function is constant only for the surface $h(r)=c r$ where $c$ is a constant. This surface is a cone and the system then reduces to

$$
\rho_{0}^{\prime}=2 \alpha-\frac{\rho_{0}+V}{r}, \quad V^{\prime}=V / r
$$

the general solution of which is

$$
V=d r, \quad \rho_{0}=\frac{1}{2}[(2 \alpha-d) r-e / r]
$$


where $d$ and $e$ are arbitrary constants, except that $e \geq 0$. It will be observed that the cone parameter $c$ does not appear in the differential equation or in the boundary conditions $T(a)=0, T(b)=p$. The solution $\rho_{0}(r)$ for given boundary conditions is, therefore, the same for all cones. In particular, if $c=0$, the cone is a plane, so that the solution for a conical membrane is the same as that for the plane membrane formed by projection of the cone on a plane perpendicular to its axis.

An interesting property of the solution for a cone is that if $V$ is to vanish for some $r \neq 0$, then $V$ is identically zero. If the deformation closes the hole so that $\rho_{0}(a)=0$ then $V(a)=0$ unless $T(a)$ is infinite. Therefore, if $T$ is to be finite and if the hole is closed, then $V=0$. In terms of the stress force $T$, this means that $T=2 \mu$ throughout the membrane.

This result is surprising because it is independent of the radius of the hole in the stress free state and of the vertex angle of the cone. It turns out that this result is even independent of the fact that the surface is a cone. The proof of this is elementary. From (3.3b), it follows that $V^{\prime}$ and $V$ have the same sign. Thus, if $V \geq 0$ for some $r$ then $V \geq 0$ for all greater $r$, and if $V \leq 0$ at some $r$ then $V \leq 0$ for all greater $r$. It follows that if $V\left(r_{0}\right)=0$ for some $r_{0}$ in $(a, b)$ then $V \equiv 0$ in $\left(a, r_{0}\right)$. Since $V$ is analytic because the right side of (17) is analytic, $V \equiv 0$ if it is zero on some interval. Finally, we must dispose of the possibility $V(a)=0$ or $V(b)=0$. To do this, it suffices to observe that for some positive $\epsilon$ the system (17) may be solved for $r$ in $[a-\epsilon, b+\epsilon]$, since a $>0$. Therefore the same argument as before holds. This shows that $V$ either has no zeroes in $[a, b]$ or is identically zero. In terms of stress force, $T=2 \mu$ throughout the membrane or $T$ has nowhere the value $2 \mu$.

This leads to an interesting geometric interpretation of Eq. (3.3a). Consider this equation in the form

$$
\left(\frac{g\left(\rho_{0}\right) d \rho}{g(r)} d r-1\right)+\left(\frac{\rho_{0}}{r}-1\right)=-\frac{2 \mu}{\lambda+2 \mu}-\frac{V}{r} .
$$

The left side is the sum of the principal extensions, so that the equation states that the sum of the principal extensions is everywhere greater than, less than, or equal to $-2 \mu /(\lambda+2 \mu)$ according to whether $T$ is less than, greater than, or equal to $2 \mu$.

The last two results give a special significance to the stress force $2 \mu$ and to the sum of principal extensions $\tau_{0}=-2 \mu /(\lambda+2 \mu)$ by showing that they are both attained either everywhere or nowhere. This is related to a result of F. John [3], which concerns plane strain problems and is valid for a more general strain energy density function than the one used in this paper (John's result holds for media of harmonic type). Specialized to the standard strain energy density the relevant results of [3] are that in the absence of body forces the stress force for plane deformation is either everywhere less $2 \mu$, everywhere greater than $2 \mu$, or everywhere equal to $2 \mu$. These three situations respectively correspond to the sum of the principal stresses being everywhere $\leq \tau_{0}, \geq \tau_{0}$, or $=\tau_{0}$. Our result in this paper does not appear to be a special case of John's, since the deformation we are considering is not a plane one even though it is two-dimensional. Nevertheless the results of [3] stated above hold. In fact, a slightly stronger result holds, for when comparing the sum of the principal strains to $\tau_{0}$, strict inequality signs hold when $V \neq 0$.

We have yet to discuss boundary conditions for the Eqs. (3.1). The condition $T(a)=0$ gives the boundary condition 


$$
V(a)=\frac{-2 \mu}{\lambda+2 \mu} \rho_{0}(a) .
$$

Let us complete the specification of boundary conditions by prescribing $\rho_{0}(a)$. By the preceding results, $V=0$, or $T=2 \mu$ when $\rho_{0}(a)=0$. Since the solution of (3.3) depends continuously on the parameter $\rho_{0}(a)$, we may conclude that as $\rho_{0}(a) \rightarrow 0$, the functions $V\left(r ; \rho_{0}(a)\right)$ approach zero uniformly, where $V\left(r ; \rho_{0}(a)\right)$ denotes the solution of (3.1) with the prescribed value of $\rho_{0}(a)$. Interpreted in terms of the stress force, this means $T\left(r ; \rho_{0}(a)\right)$ approaches the function which is zero at $r=a$ and is $2 \mu$ elsewhere when $\rho_{0}(a) \rightarrow 0$. Finally, we may, as the second boundary condition, prescribe $p=T(b)$ instead of $\rho_{0}(a)$. From the above results it follows that $\rho_{0}(a) \rightarrow 0$ may in all cases be replaced by $p \rightarrow 2 \mu$.

The foregoing results were tested for a special case in the course of the numerical work to be described later, a spherical surface being considered:

$$
\begin{gathered}
h(r)=1-\left(1-r^{2}\right)^{1 / 2}, \\
a=0.4, \quad b=0.9, \quad \mu=0.5, \quad \lambda=0.75 .
\end{gathered}
$$

The results are shown in Fig. 2.

4. Perturbed equations. To determine the buckling parameters we now perturb the equilibrium equations (2.5) about the symmetric solution $\rho_{0}(r)$. We suppose that, for all sufficiently small $\epsilon$, there exist solutions of the equilibrium equations of the form (1.5) and (1.6) satisfying the boundary conditions (2.6). We substitute (1.5) and (1.6) into (2.5) and (2.6) and differentiate the resulting equations with respect to $\epsilon$. Finally, we evaluate the differentiated equations at $\epsilon=0$, obtaining

$$
\begin{aligned}
A_{1} \eta_{r r}+A_{2} \eta_{\theta \theta}+A_{3} \psi_{r \theta}+A_{4} \eta_{r}+A_{5} \psi_{\theta}+A_{6} \eta=0 \\
B_{1} \psi_{r r}+B_{2} \psi_{\theta \theta}+B_{3} \eta_{\theta r}+B_{4} \eta_{\theta}+\dot{B}_{5} \psi_{r}=0
\end{aligned}
$$

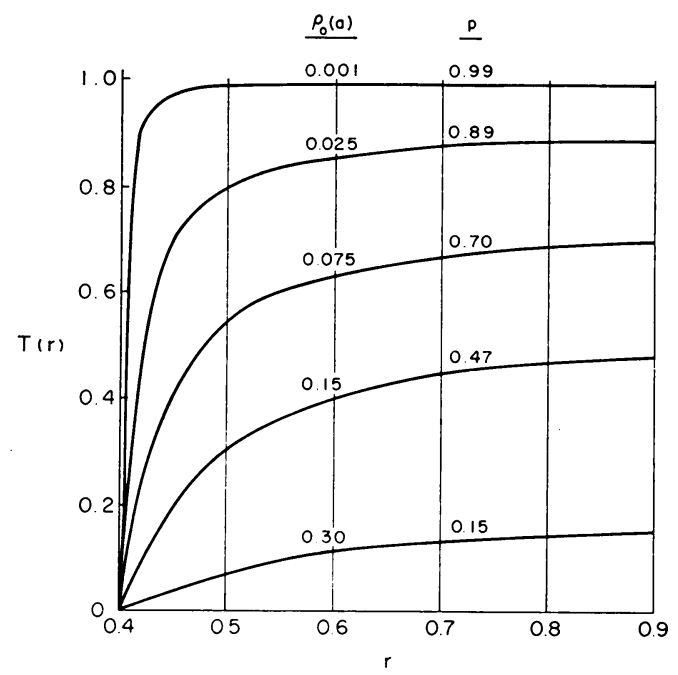

Fig. 2. Stress force $\mathrm{T}(r)$ versus $r$ for different pressures calculated for a membrane on a sphere of radius 1 with $a=0.4, b=0.9, \lambda=0.75, \mu=0.5$. 


$$
\left.\begin{array}{r}
\eta\left[p \rho_{0} g_{0}^{\prime}+p g_{0}+k_{1}\right]+k_{2} \eta_{r}+\left[p \rho_{0} g_{0}+k_{3}\right] \psi_{\theta}=0, \\
{\left[p \rho_{0} g_{0}-m_{1} \eta_{\theta}\right]-m_{2} \psi_{r}=0,}
\end{array}\right\} \text { at } r=b,
$$

Expressions for the values $A, B, k$ and $m$ are given in Appendix 2. These coefficients may be expressed in terms of $r, \rho_{0}(r)$ and $\rho_{0}^{\prime}(r)$. Since $\rho_{0}$ is determined by $p$, the coefficients may be considered as functions of $r$ which depend also on a parameter $p$.

Next, we substitute the Fourier series (1.9) into the system (4.1) with boundary conditions (4.2). For each positive integer $n$, we obtain two systems of second-order differential equations. One system is of the form

$$
F_{1}\left(a_{n}^{*}, b_{n}\right)=F_{2}\left(a_{n}^{*}, b_{n}\right)=0,
$$

where $F_{1}$ and $F_{2}$ are differential operators, while the other is of the form

$$
F_{1}\left(a_{n}, b_{n}^{*}\right)=F_{2}\left(a_{n}, b_{n}^{*}\right)=0
$$

with the same operators $F_{1}$ and $F_{2}$. It therefore suffices to solve the system $F_{1}(x, y)=$ $F_{2}(x, y)=0$. The boundary conditions separate similarly. Explicitly, the differential equations and boundary conditions are

$$
\left.\begin{array}{rl}
A_{1} x^{\prime \prime}+A_{4} x^{\prime}+n A_{3} y^{\prime}+\left(A_{6}-n^{2} A_{2}\right) x+n A_{5} y, & =0, \\
B_{1} y^{\prime \prime}+B_{5} y^{\prime}-n^{2} B_{2} y-n B_{3} x^{\prime}-n B_{4} x & =0, \\
\left(p \rho_{0} g_{0}^{\prime}+p g_{0}+k_{1}\right) x+k_{2} x^{\prime}+\left(p \rho_{0} g_{0}+k_{3}\right) n y & =0, \\
\left(n \rho_{0} g_{0}-m_{1}\right) n x+m_{2} y^{\prime} & =0,
\end{array}\right\} \text { at } r=\mathrm{b},
$$

The $n$th critical pressure $p_{n}$ is the eigenvalue of this system.

5. Numerical solution. To compute the critical pressures for a given membrane we must resort to numerical techniques. We describe briefly a numerical method for determining the eigenvalue of the system (4.3) with boundary conditions (4.4). The method is based on the following observations: let $x(r)$ and $y(r)$ be a nontrivial solution of the perturbed equations which satisfies the boundary conditions. If it happened that $x(a)$ and $x^{\prime}(a)$ were both zero then the last two of the four boundary conditions would imply (barring the vanishing of certain coefficients) that $y(a)=y^{\prime}(a)=0$, which would give $x(r)=y(r)=0$ for all $r$. Therefore we may suppose that $x(a)$ and $x^{\prime}(a)$ are not both zero. Furthermore, since both the differential equations and the boundary conditions are linear and homogeneous, we may normalize the solution by multiplying it by a suitable constant. From this it follows that it suffices to look for solutions satisfying the condition $x^{\prime}(a)=\left[1-x^{2}(a)\right]^{1 / 2}$.

Using this fact, we may construct a numerical scheme for finding the critical pressure. For a given value of $p$ and $x(a)$ satisfying $-1 \leq x(a) \leq 1$ we may find out as follows, how close $p$ and $x(a)$ come to being the critical pressure and the value for which the perturbed system has a solution satisfying $x^{\prime}(a)=\left[1-x^{2}(a)\right]^{1 / 2}$. For the given $p$ we solve the equilibrium Eqs. (3.3) for the symmetric deformation and, using the values 
of $\rho_{0}(r)$ and $\rho_{0}^{\prime}(r)$ obtained from this solution, compute the coefficients of the perturbed equations and boundary conditions. (The former vary with $r$, therefore values of these coefficients or of $\rho_{0}$ and $\rho_{0}^{\prime}$ must be tabulated.) Knowing the values of $x(a)$ and $x^{\prime}(a)$, we compute from the last two of the four boundary conditions the values of $y(a)$ and $y^{\prime}(a)$. This gives initial data for the solution of the perturbed linear system, which is then solved. The resulting solution automatically satisfies the last two boundary conditions. To see if it satisfies the remaining boundary conditions $(4,4 a, b)$, we evaluate the expressions on the left sides of these conditions. We denote them by $B_{i}(p, x(a)),(i=1,2)$. $p$ is a critical pressure if $B_{1}=B_{2}=0$. To find a critical pressure we thus must find the common roots of the functions $B_{1}$ and $B_{2}$. Since we can evaluate these functions we may find their common roots by any one of several standard methods.

The scheme outlined above was programmed for and run on the IBM 7090 at New York University. Instead of using the parameter $p$, as described above, we used the critical value of $\rho_{0}(a)$, for with a prescribed value of $\rho_{0}(a)$ solution of the system (3.3) for the symmetric deformation is an ordinary initial value problem, while with $p$ prescribed it is a two point boundary value problem. The $p$ corresponding to a given $\rho_{0}(a)$ is computed in the course of solving (3.3) for the symmetric deformation, so that the critical pressure is found also when the critical value of $\rho_{0}(a)$ is computed. The RungeKutta method was used to integrate the systems of ordinary differential equations. The common roots of $B_{1}$ and $B_{2}$ were found by the Newton-Raphson method, using central difference approximations for the required derivatives.

The program was tested for on a plane membrane, because the value of the critical pressure may then be compared with that given by the formula of Sensenig [2]. For the plane membrane with the parameters

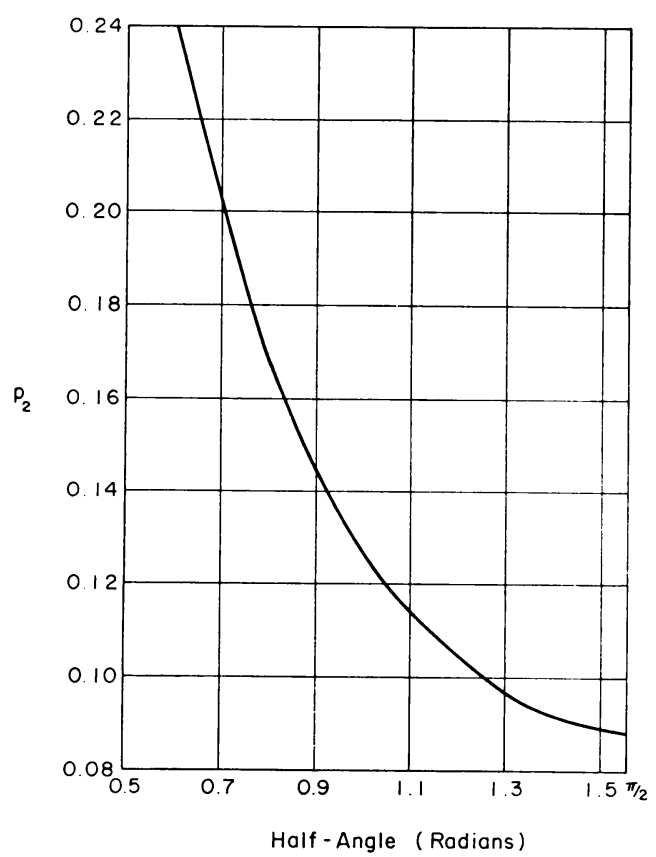

FIG. 3. Buckling pressure $p_{2}$ in second mode for membrane on a cone of varying half-angle. Membrane parameters: $a=0.50, b=1.00, \lambda=0.75, \mu=0.5$. 


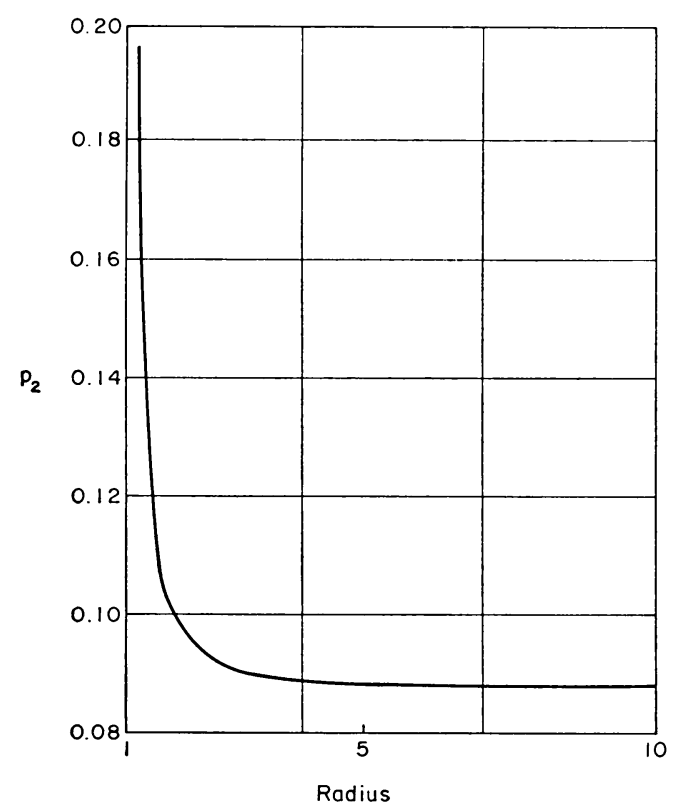

FIG. 4. Buckling pressure $p_{2}$ in second mode for membrane on a sphere of varying radius. Membrane parameters: $a=0.50, b=1.00, \lambda=0.75, \mu=0.5$.

$$
a=0.5, \quad b=1.0, \quad=0.75, \quad=0.5
$$

Sensenig's formula and our program gave, to five places, the same values for $p_{2}$ and $p_{3}$.

Next, the program was used to study the second critical pressure of a membrane on a sphere of varying radius and on a cone of varying half angle. The membrane parameters used were the same as those of the plane membrane above. For a sphere of large radius and for a cone of half angle near $\pi / 2$ the computed values of $p_{2}$ were very near the value of $p_{2}$ for a plane membrane, as might be expected. Results of the sphere and cone calculations are shown in Figs. 3 and 4.

Acknowledgment. The author wishes to thank Professors E. Isaacson and J. J. Stoker of New York University for their advice and encouragement.

\section{Appendix I}

Equations (2.2) through (2.5) give $W$ in terms of $p, \rho_{r}, \rho_{\theta}, \phi_{r}$ and $\phi_{\theta}$, and the derivatives of $W$ with respect to these quantities may be computed from these equations. A lengthy but straightforward computation gives expressions for these derivatives evaluated at $\rho(r, \theta)=\rho_{0}(r), \phi(r, \theta)=\theta$. We list the necessary formulas below, introducing the notation $g_{0}=g\left(\rho_{0}\right), g_{0}^{\prime}=g^{\prime}\left(\rho_{0}\right), g_{0}^{\prime \prime}=g^{\prime \prime}\left(\rho_{0}\right)$. Superscript zero denotes evaluation for the symmetric deformation. Derivatives which are identically zero are omitted.

$$
\begin{aligned}
& r g(r) W_{\rho}^{0}=(2 \mu+\lambda)\left[\frac{r g_{0} g_{0}^{\prime}\left(\rho_{0}^{\prime}\right)^{2}}{g(r)}+\frac{\rho_{0} g(r)}{r}\right]+\lambda \rho_{0}^{\prime}\left[\rho_{0} g_{0}^{\prime}+g_{0}\right]-2(\mu+\lambda)\left[r \rho_{0}^{\prime} g_{0}^{\prime}+g(r)\right], \\
& r g(r) W_{\rho_{r}}^{0}=(2 \mu+\lambda) \frac{r \rho_{0}^{\prime} g_{0}^{2}}{g(r)}+\lambda \rho_{0} g_{0}-2(\mu+\lambda) r g_{0},
\end{aligned}
$$




$$
\begin{aligned}
& r g(r) W_{\phi \theta}^{0}=(2 \mu+\lambda) \frac{\rho_{0}^{2} g(r)}{r}+\lambda \rho_{0} g_{0} \rho_{0}^{\prime}-2(\mu+\lambda) \rho_{0} g(r) \\
& r g(r) W_{\rho \rho}^{0}=(2 \mu+\lambda) \frac{g(r)}{r}+\frac{r\left(\rho_{0}^{\prime}\right)^{2}}{g(r)}\left[\left(g_{0}^{\prime}\right)^{2}+g_{0}^{\prime} g_{0}^{\prime \prime}\right]+\lambda \rho_{0}^{\prime}\left[\rho_{0} g_{0}^{\prime \prime}+2 g_{0}^{\prime}\right]-2(\mu+\lambda) r \rho_{0}^{\prime} g_{0}^{\prime \prime}, \\
& r g(r) W_{\rho \rho_{r}}^{0}=2(2 \mu+\lambda) \frac{r \rho_{0}^{\prime} g_{0} g_{0}^{\prime}}{g(r)}+\lambda\left[g_{0}+\rho_{0} g_{0}^{\prime}\right]-2(\mu+\lambda) r g_{0}^{\prime} \\
& r g(r) W_{\rho \phi \theta}^{\theta}=2(2 \mu+\lambda) \frac{\rho_{0} g(r)}{r}+\lambda \rho_{0}^{\prime}\left[\rho_{0} g_{0}^{\prime}+g_{0}\right]-2(\mu+\lambda) g(r) \\
& r g(r) W_{\rho_{r} \rho_{r}}^{0}=(2 \mu+\lambda) \frac{r g_{0}^{2}}{g(r)}, r g(r) W_{\rho_{r} \phi \theta}^{0}=\lambda \rho_{0} g_{0} \\
& r g(r) W_{\rho \theta \rho \theta}^{0}=(2 \mu+\lambda) \frac{g_{0}^{2} g(r)}{r}-2(\mu+\lambda) \frac{\left[g_{n} g(r)\right]^{2}}{r \rho^{\prime} g_{0}+\rho_{0} g(r)} \\
& r g(r) W_{\rho \theta \phi r}^{0}=-\lambda \rho_{0} g_{0}+2(\mu+\lambda) \frac{r g_{0} \rho_{0} g(r)}{r \rho^{\prime} g_{0}+\rho_{0} g(r)}, \\
& r g(r) W_{\phi r \phi r}^{0}=(2 \mu+\lambda) \frac{r \rho_{0}^{2}}{g(r)}-2(\mu+\lambda) \frac{\left(r \rho_{0}\right)^{2}}{r \rho_{0}^{\prime} g_{0}+\rho g(r)} \\
& r g(r) W_{\phi \theta \phi \theta}^{0}=(2 \mu+\lambda) \frac{\rho_{0}^{2} g(r)}{r} .
\end{aligned}
$$

Appendix II

We list here the coefficients in the perturbed equations (4.3) and (4.4). The following new notation is used: if $x$ and $y$ are any two of the arguments of $W$,

$$
W_{x y}^{\prime}=r g(r) W_{x y}^{0}
$$

With this notation,

$$
\begin{aligned}
& A_{1}=W_{\rho_{r} \rho_{r}}^{\prime}, \quad B_{3}=W_{\rho_{r} \phi \theta}^{\prime}+W_{\rho_{\theta \phi r}}^{\prime}, \\
& A_{2}=W_{\rho \theta \rho \theta}^{\prime}, \quad B_{4}=W_{\rho \phi \theta}^{\prime}+\frac{d}{d r} W_{\rho \theta \phi r}^{\prime}, \\
& A_{3}=W_{\rho_{r} \phi \theta}^{\prime}+W_{\rho_{\theta \phi_{r}}}^{\prime}, \quad B_{5}=\frac{d}{d r} W_{\phi_{r} \phi_{r}}^{\prime}, \\
& A_{4}=\frac{d}{d r} W_{\rho_{r} \rho_{r}}^{\prime}, \quad k_{1}=W_{\rho \rho_{r}}^{\prime}, \\
& A_{5}=\frac{d}{d r} W_{\rho \rho_{r}}^{\prime}-W_{\rho \rho}^{\prime}, \quad k_{2}=W_{\rho_{r} \rho_{r}}^{\prime}, \\
& A_{6}=\frac{d}{d r} W_{\rho \rho r}^{\prime}-W_{\rho \rho}^{\prime}, \quad k_{3}=W_{\rho_{r} \phi \theta}^{\prime}, \\
& B_{1}=W_{\phi_{r} \phi_{r}}^{\prime}, \quad m_{1}=W_{\rho \theta \phi_{r}}^{\prime}, \\
& B_{2}=W_{\phi_{\theta \phi \theta}}^{\prime}, \quad m_{2}=W_{\phi_{r} \phi_{r}}^{\prime} \text {. }
\end{aligned}
$$




\section{REFERENCES}

1. S. Lubkin, Determination of buckling criteria by minimization of total energy, New York Univ., Institute of Math. Sciences, Report No. 241, 1957

2. C. Sensenig, Instability of thick elastic solids, New York Univ., Courant Institute of Math. Sciences, Report No. 310, 1963

3. F. John, Plane strain problems for a perfectly elastic material of harmonic type, Comm. Pure Appl. Math. 13 (1960) 